EPJ Web of Conferences 87,03001 (2015)

DOI: $10.1051 /$ epjconf/ 20158703001

(C) Owned by the authors, published by EDP Sciences, 2015

\title{
Summary of ECE Presentations at EC-18
}

\author{
G. Taylor ${ }^{1, a}$ \\ ${ }^{1}$ Princeton Plasma Physics Laboratory, Princeton, NJ 08543, USA
}

\begin{abstract}
There were nine ECE and one EBE presentation at EC-18. Four of the presentations were on various aspects of ECE on ITER. The ITER ECE diagnostic has entered an important detailed preliminary design phase and faces several design challenges in the next 2-3 years. Most of the other ECE presentations at the workshop were focused on applications of ECE diagnostics to plasma measurements, rather than improvements in technology, although it was apparent that heterodyne receiver technology continues to improve. CECE, ECE imaging and EBE imaging are increasingly providing valuable insights into plasma behavior that is important to understand if future burning plasma devices, such as ITER, FNSF and DEMO, are to be successful.
\end{abstract}

\section{ECE on ITER}

There were four presentations on ITER ECE at the EC-18 workshop. A session with three oral presentations and a discussion were devoted to this subject. An invited talk (G. Taylor) was presented on the current status of the design of the ITER ECE diagnostic that is being developed in a collaborative effort between India, the United States and the ITER central team. A detailed preliminary design phase has begun and will be completed in late 2015. The final design will be reviewed about a year later. Although there are spatial contraints in the front-end of the diagnostic due to the need for significant shielding to protect components, the design continues to include both radial and oblique plasma views and two hot calibration sources.

Advances in technology will allow significant improvements to the ITER ECE instrumentation in the coming years. The heterodyne radiometer systems will use robust, highly-integrated, front-end designs that operate up to $350 \mathrm{GHz}$. Advances in high-speed digital circuitry will allow direct digitization of IF signals well into the GHz range. Direct digitization of ECE using high $\mathrm{T}_{\mathrm{c}}$ Josephson junction detection and Hilbert-transform spectral analysis may allow improved signal-to-noise and reduced calibration times below $350 \mathrm{GHz}(\mathrm{H}$. K. B. Pandya).

Recent extensive ECE simulations performed for the ITER ECE diagnostic are in good agreement with previous studies [P. V. Subhash]. Good radial resolution for both plasma views was confirmed, with the oblique view generally having better resolution, but reduced radial coverage. The oblique view is sensitive to even a small population of non-thermal electrons.
The ITER ECE discussion raised several concerns about the present design. In the current design the oblique view is pointing towards an ECH launcher and the coherent Thomson scattering (CTS) system, that uses megawatt-level $60 \mathrm{GHz}$ gyrotrons. It may be possible to flip the oblique view to the other side, but this is probably not trivial. The audience encouraged the designers to consider transmission line options other than smooth metal walled waveguide, and to move the splitter boxes further away from the machine.

A poster on the challenging maintenance environment for the ITER ECE front-end components was presented by V. Udinstev. A significant effort is being made to protect workers from radiation exposure, by using assisted-manual tools for handling equipment near the port plug and robotics tools deployed in the Hot Cell Facility, where a human presence is not planned. A significant effort is also being made to prevent tritium and beryllium contamination in the port cell near the machine.

\section{ECE measurements}

2-D ECE imaging has been used to study multiple flux tubes driven by ECH/ECCD in sawtoothing KSTAR plasmas (G. Yun). An example was shown where ECH was deposited inside the sawtooth inversion radius. Clear images of flux tube evolution from triple to dual to single flux tubes were obtained. The measurements were found to be in good agreement with non-linear MHD simulations suggesting that the q-profile was flat after the sawtooth crash and that there was growth and saturation of $\mathrm{m} / \mathrm{n}=1 / 1$ helicity flux tubes generated by the ECH that eventually merged into a single $m=1$ mode.

\footnotetext{
${ }^{\mathrm{a}}$ Corresponding author: gtaylor@pppl.gov
} 
Detection of NTMs before the mode size becomes large is important for effective stabilization with ECH. ECE measurements inline with an ECH launcher on ASDEX-U confirm the presence of a small NTM in a Fourier transform of the ECE data (H. van den Brand). But the signal-to-noise ratio was small and there are plans to increase the signal-to-noise ratio by averaging and hardware improvements. Another approach being pursued is to control the sawtooth instability to prevent NTMs in the first place by using a real-time sawtooth crash detection algorithm. This method detects the sawtooth crash by sampling at $1 \mathrm{MHz}$ and using a tension spline fit derivative analysis.

On C-Mod correlation ECE (CECE) measured reduced $\mathrm{T}_{\mathrm{e}}$ fluctuations across the transition from the linear ohmic confinement (LOC) regime to the saturated ohmic confinement (SOC) regime (C. Sung). Reduction in $\mathrm{T}_{\mathrm{e}}$ fluctuations at $\rho \sim 0.8$ was observed across the LOC/SOC transition in both ohmically-heated and RFheated plasmas. Gyrokinetic simulations showed no change in the dominant turbulence mode across the LOC/SOC transition. These results are contrary to the conventional interpretation that the saturation in ohmic confinement is due to a transition from a TEM-dominated to ITG-dominated fluctuation regime. Local changes in turbulence will need to be studied in more detail in the future in order to better understand the physics underlying the transition from the LOC to the SOC regime.

A new high-resolution 8-channel heterodyne radiometer with a radial resolution $\sim 8 \mathrm{~mm}$ has been installed on DIII-D. It has been used to detect fine structure in MHD modes (M. Austin). An example was shown where the phase inversion radius of a slowly rotating, nearly locked, mode was resolved to less than a $\mathrm{cm}$.

Digital CECE has been implemented for turbulence measurements on LHD (H. Tsuchiya). The instrument combines direct IF band digitization and CECE. Direct IF digitization is a technique that allows the choice of the spatial and temporal resolution after data acquisition. The instrument used an $80 \mathrm{GHz}$ sampling ADC, but concerns were raised that the ADC had an insufficient (8-bit) resolution depth.

\section{EBE measurements}

There was one paper presented on EBE imaging at the workshop. Microwave imaging measured $\mu$ s EBE bursts correlated with ELMs from overdense plasmas in MAST (S. Freethy). The bursts occur at the midplane $20 \mu \mathrm{s}$ before the D-alpha peak and there were many bursts at each ELM. It appears that the origin of the EBE is Doppler-broadened second harmonic emission originating from MHD-generated fast electrons. The imaging revealed that the bursts are highly spatially localized. However it was not clear why the emission was asymmetric and localized near the midplane. The ELMrelated EBE bursts on MAST appear to be similar to
ELM-related ECE bursts observed previously on underdense tokamaks (eg. TFTR, DIII-D).

\section{Concluding remarks}

CECE, ECE imaging and EBE imaging are increasingly providing valuable insights into plasma behavior that needs to be better understood for successful operation of future fusion devices, such as ITER, FNSF and DEMO. As the detailed design of the ITER ECE diagnostic begins there are still several design challenges that need to be resolved. This diagnostic system will be critical to the success of the ITER mission because of its ability to measure the alpha particle heating profile, detect NTMs and measure ECE power loss and transport. There were notably only brief discussions at the workshop related to the long-standing ECE/Thomson scattering $T_{e}$ discrepancy previously observed on TFTR and JET. However understanding this discrepancy remains an important potential issue for ITER. 